\title{
Chaotic Motions In the Dynamics of a Hopping Robot
}

\author{
A.F. Vakakis, J.W. Burdick \\ School of Engineering and Applied Science \\ Mail Code 104-44 \\ California Institute of Technology, Pasadena, CA 91125
}

\begin{abstract}
This paper applies discrete dynamical systems theory to the dynamic stability analysis of a simplified hopping robot. A Poincaré return map is developed to capture the system dynamic behavior, and two basic non-dimensional parameters which influence the systems dynamics are identified. The hopping behavior of the system is investigated by constructing the bifurcation diagrams of the Poincare return map with respect to these parameters. The bifurcation diagrams show a period doubling cascade leading to a regime of chaotic behavior, where a "strange attractor" is devel oped. An interesting feature of the dynamics is that the strange attractor can be controlled and eliminated by tuning an appropriate parameter corresponding to the duration of applied hopping thrust. Physically, the collapse of the strange attractor leads to globally stable uniform hopping motion.
\end{abstract}

\section{Introduction}

In his pioneering work [1], Raibert has examined theoretical and experimental models of one-legged and multi-legged hopping robots. He used a variety of control procedures to achieve a stable hopping motion, i.e., a steady state motion which repeats itself after each hop. In order to understand some of the interesting dynamical behavior seen in Raibert's experiments, Koditschek and Bühler have applied discrete dynamical systems theory to study the dynamics of a simplified one-legged hopping robot model [2]. Their simplified model includes only the vertical robot hopping motion, but uses a control system analogous to that used by Raibert in his experiments. In particular, Koditschek and Bühler showed that period-1 hopping motions are not the only possible stable hopping gaits. They analytically derived conditions for stable period-2 hopping orbits, which may correspond to the "limping gaits" which were experimentally observed by Raibert.

This work extends the analysis in [2]. The dynamical analysis in this work, as well as in [2], is based on the development of a Poincare return map [3] of the hopping robot dynamics. We derive a more complete model of the system studied by Koditschek and Bühler in which the assumption of instantaneous thrust $d u-$ ration made in [2] is relaxed. The rich dynamics of this system are studied by means of the bifurcation diagrams of the return map. We show that some of the assumptions used in deriving the return map in [2] lead to a structurally unstable return map, and that the more realistic model used in this work leads to qualitatively different and interesting global dynamics of the hopping system. The dynamics of the instantaneous thrust model exhibit the classic period doubling route to chaos and the existence of a strange attractor. In the more complete model derived in this paper, it is shown that for finite time of thrust application, the structure of the strange attractor becomes less complex. For sufficiently large time of thrust, the strange attractor totally collapses, and the robot has only a stable period one hopping motion. In other words, by proper choice of control parameters, the robot will exhibit a globally stable uniform hopping motion for a large range of the physical model parameters.

The structure of this paper is as follows. In Section 2 the simplified hopping robot dynamic model is derived. This model is used in Section 3 to derive the Poincaré return map. Section 4 investigates the bifurcation behavior of this map with respect to the physical system and control parameters. In Section 5 the hopping robot dynamics are simulated to verify some of the bifurcation diagram predictions made in Section 4. A more complete analysis and set of simulations can be found in [7], and more advanced analysis of the map will be reported in an upcoming report [8].

\section{Description of the Hopping Robot Model}

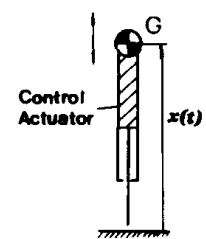

Figure 1: Simplified hopping robot model

The simplificd hopping robot appcars in Figure 1 (this model follows closely the notation used in [2], and [2] should be consulted for more details of the physical reasoning used to derive this model). The total mass of the system is concentrated at point G, and is assumed, without loss of generality to be equal to a unit mass. The energy storage mechanism (stiffness) of the leg is a pneumatic cylinder and hence the restoring force is of the form:

$$
F_{s}=\phi(x)=\frac{1}{x}
$$

The pneumatic cylinder is controlled by an adjustable stiffness, $\eta(x, \dot{x}, t)$, which multiplies the stiffness force $F_{s}$.

The motion and control of the robot can be divided into four phases, as shown in Figure 2:

i. Thrust. Assuming that at time $t_{j}$ the center of mass of the robot is at its minimum height, $x_{j}=x\left(t_{j}\right)$ (i.e., $\dot{x}\left(t_{j}\right)=0$ ), the control valves are opened and an constant supply pressure is connected to the leg cylinder for a fixed time, $\delta_{t}$. This results in a constant thrust force, $\tau$, which is the product of the supply pressure and pneumatic cylinder cross section. 


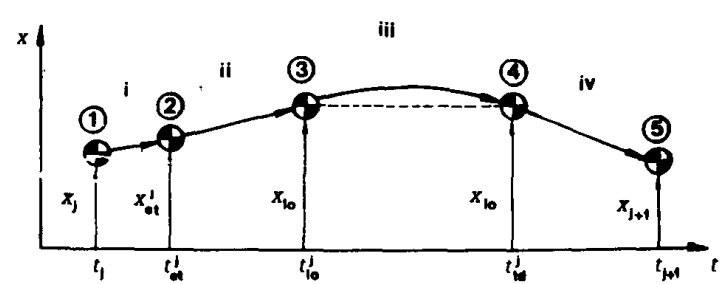

Figure 2: Four phases of hopping motion. During this phase the equation of motion of point $G$ is:

$$
\ddot{x}-\tau+g=0 \quad \text { for } t_{j} \leq t \leq t_{j}+\delta_{t}
$$

where $g$ is the gravitational constant. Note that the fixed thrust $\tau$ may be less than the effective thrust built up in the cylinder due to leg compression, and energy loss can occur in this cycle.

ii. Decompression. At the end of the thrust phase, the valves are closed, defining an effective spring constant, $\eta_{2} . \eta_{2}$ is a function of the body position at the end of the thrust phase, $x_{e t}^{j}$, and is given by $\eta_{2}=\tau x_{e t}^{j}$. The equation of motion during this phase is:

$$
\ddot{x}-\frac{\tau x_{e t}^{j}}{x}+g=0 \quad x_{e t}^{j} \leq x \leq x_{l_{0}}
$$

where $x_{l_{0}}$ is the uncompressed length of the pneumatic cylinder. When the height $x$ reaches $x_{l_{0}}$, the robot loses contact with the ground.

iii. Flight. For this phase, we assume that friction induced by air drag during flight is negligible and the robot's energy is conserved. Consequently, the lift-off height $x_{l_{0}}$ is identical to the touchdown height. The equation of motion in this phase is:

$$
\ddot{x}+g=0 \quad x>x_{l_{0}}
$$

iv. Compression Phase. At touchdown, $\left(t_{t d}^{j}, x_{l_{0}}\right)$, an initial pressure exists in the leg, fixing the spring constant during compression at $\eta_{1} . \eta_{1}$ is assumed to be uniform during every compression phase, and the leg pressure is adjusted during the flight phase if necessary. The equation of motion during this phase is:

$$
\ddot{x}-\frac{\eta_{1}}{x}+g=0
$$

At the end of this phase, a new minimum height $x_{j+1}$ is reached at time $t_{j+1}$, and a new hopping cycle starts.

An elastic impact of the robot with the rigid ground is assumed so that there are no frictional losses in any phase of the motion.

\section{Derivation of the Poincaré Return Map}

Equations (2)-(5) determine the motion of this dynamical system through four subsequent phases of a complete hopping cycle, and the phase space of the system is 3-dimensional: $(x, \dot{x}, t)$. Figure 3 shows the phase space during one hopping cycle. Let $\sum$ be the phase plane surface, or "Poincaré section," such that $\dot{x}=0$. The trajectory will pierce $\sum$ at two distinct points, $A$ and $A^{\prime}$, provided the following transversality condition is satisfied at the points of intersection:

$$
(\dot{x}, \ddot{x}, t) \cdot(0,1,0) \neq 0 \Rightarrow \ddot{x} \neq 0
$$

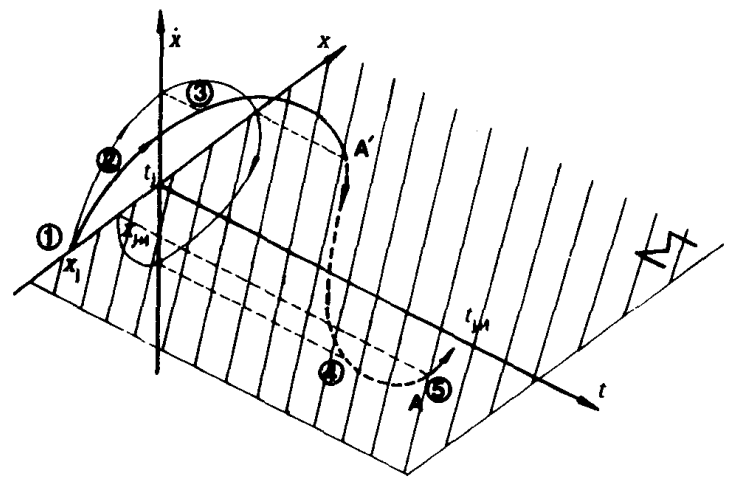

Figure 3: Phase space and Poincaré section

$A^{\prime}$ corresponds to the maximum height attained by the robot, and $A$ corresponds to the minimum height which is also the point and $A$ corresponds to the minimum height which a Poincaré Return Map [3] can be defined as:

$$
P: \sum \rightarrow \sum:\left(\begin{array}{c}
x_{j} \\
t_{j}
\end{array}\right) \stackrel{P}{\rightarrow}\left(\begin{array}{c}
x_{j+1} \\
t_{j+1}
\end{array}\right)
$$

or

$$
x_{j+1}=f\left(x_{j}, t_{j}\right) \quad t_{j+1}=g\left(x_{j}, t_{j}\right)
$$

The form of the functions $f(\cdot, \cdot)$ and $g(\cdot, \cdot)$, if they exist in analytical form, is determined by the flow of the dynamical system and the position of the Poincaré Section, $\sum$.

Hence, we have converted the study of the dynamics of the hopping robot to the study of the nonlinear mapping (7). The fixed points, or period 1 orbits, of (7) correspond to period-one hopping in which the robot's hop is uniform from bounce to bounce. Similarly, a period-m orbit of (7) corresponds to a robot motion which repeats itself after $m$ hops. The stability of periodic motions of the continuous dynamical system is determined completely by the stability of the periodic orbits of the mapping (7).

In deriving the explicit form of the Poincaré map, we assume that gravity forces during the stance phases of motion are negligible compared to the thrust and stiffness forces. This same assumption was made in [2], and the effects of including gravity during the stance phase will be discussed below. The detailed derivation of the return map can be found in [7]. In brief, the return map is determined by integrating equations (2)-(5) over their respective motion phases, using as initial conditions the final conditions of the previous phase. If $t_{j}$ denotes the time at which the robot reaches maximum compression $x_{j}$ (the beginning of a cycle), the state of the robot at the beginning of the next cycle (next maximum compression) is:

$$
\begin{array}{r}
x_{j+1}=x_{l_{0} e}\left[\frac{-\tau^{2} \delta_{t}^{2}+2 \tau\left(\frac{1}{2} \tau \delta_{t}^{2}+x_{j}\right) \ln \left(\frac{x_{l_{0}}}{\left.\frac{1}{2 \tau \delta_{t}^{2}+x_{j}}\right)}\right)}{2 \eta_{1}}\right] \\
t_{j+1}=\delta_{t}+t_{j}+\frac{2\left[\tau^{2} \delta_{t}^{2}+2 \tau x_{e t}^{j} \ln \left(\frac{x_{l_{0}}}{x_{e t}^{j}}\right)\right]^{\frac{1}{2}}}{g} \\
+\int_{x_{e t}^{j}}^{x_{l_{0}}} \frac{d \xi}{\left[\tau^{2} \delta_{t}^{2}+2 \tau x_{e t}^{j} \ln \left(\frac{\xi}{x_{e t}^{j}}\right)\right]^{\frac{1}{2}}}
\end{array}
$$




$$
\begin{gathered}
-\int_{x_{l_{0}}}^{x_{j+1}} \frac{d \xi}{\left[\tau^{2} \delta_{t}^{2}+2 \tau x_{e t}^{j} \ln \left(\frac{x_{l_{0}}}{x_{e t}^{j}}\right)+2 \eta_{1} \ln \left(\frac{\xi}{x_{l_{0}}}\right)\right]^{\frac{1}{2}}} \\
\dot{x}\left(t_{j+1}\right)=0
\end{gathered}
$$

Equations (8) and (9) are of the form

$$
x_{j+1}=f\left(x_{j}\right) ; \quad t_{j+1}=g\left(x_{j}, t_{j}\right) .
$$

and therefore define the mapping ( 7 ) with the assumptions stated above. Consequently, the mapping which relates two subsequent minimum heights of the robot is time-independent and therefore 1-dimensional and the complete motion of this simplified hopping robot can be studied by analyzing this 1 -dimensional map. The associated periods of the orbits can be computed using the second of equations (11). The uncoupling of time in the first of equations (11) is not related to the simplification of omitting gravity during the stance phase. If gravity is included in the stance phase, it can be shown [7] that equation (8) takes the form:

$$
\begin{gathered}
(\tau-g)^{2} \delta_{t}^{2}-2 g\left(x_{l_{0}}-X\right)+2 \tau X \ln \left(\frac{x_{l_{0}}}{X}\right) \\
=2 \eta_{1} \ln \left(\frac{x_{j+1}}{x_{l_{0}}}\right)-2 g\left(x_{j+1}-x_{l_{0}}\right)
\end{gathered}
$$

where $X=x_{j}+\frac{1}{2}(\tau-g) \delta_{t}^{2}$. Equation (12), which relates $x_{j+1}$ to $x_{j}$, is one-dimensional and time-independent. Unfortunately, (12) is transcendental, and no closed form expression of the form $x_{j+1}=f\left(x_{j}\right)$ exists, and numerical methods must be used to analyze this "complete" system [7].

\section{The Mapping Bifurcation Diagrams}

Introducing the nondimensional variables and parameters:

$$
w_{j}=\frac{x_{j}}{x_{l_{0}}} ; \quad \lambda=\frac{\tau x_{l_{0}}}{\eta_{1}} ; \quad \beta=\frac{\tau \delta_{i}^{2}}{2 x_{l_{0}}}
$$

map (8) takes the form:

$$
w_{j+1}=e^{\left\{-\lambda \beta+\left(\lambda \beta+\lambda w_{j}\right) \ln \left(\beta+w_{j}\right)\right\}}=e^{-\lambda \beta}\left(\beta+w_{j}\right)^{\lambda\left(\beta+w_{j}\right)}
$$

Since the minimum height $x_{j}$ must be always less than the uncompressed leg length, $x_{l_{0}}, w_{j}$ is physically restricted to the domain $0<w_{j}<1$. Also note that $\beta$ and $\lambda$ are a positive quantities. Graphical representations of the map (14) in the range of interest are presented in Figures $4(\mathrm{a}, \mathrm{b})$ for $\beta=0, \beta=0.1$, and various values of the parameter $\lambda$. Since $\lambda$ can become large, we consider its inverse, $\lambda^{-1}$, in the graphs (so that $\left.\lambda^{-1} \in(0,1]\right)$. As will be seen, the behavior of the map for $\beta$ equal to zero is qualitatively different from that corresponding to $\beta>0$ and hence we will examine these two cases separately. In [2], Koditschek and $B$ ühler assumed that $\delta_{t}=0$, and their work represents the study of the map (14) corresponding to $\beta=0$.

4.1. The Case $\beta=0$ (The Koditschek-Bühler Map)

Setting $\beta=0,(14)$ assumes the form:

$$
w_{j+1}=e^{\left(\lambda w_{j} \ln \left(w_{j}\right)\right)}=f\left(w_{j} ; \lambda\right)
$$

The map is defined for $w_{j}>0$ only and is singular at $w_{j}=0$. Note that $\lim _{w_{j} \rightarrow 0} w_{j+1}=1$ and the critical point (which is at a minimum) occurs at $w_{c}=1 / e$.

The fixed points (period 1 orbits), $\bar{w}$, of (15) can analytically

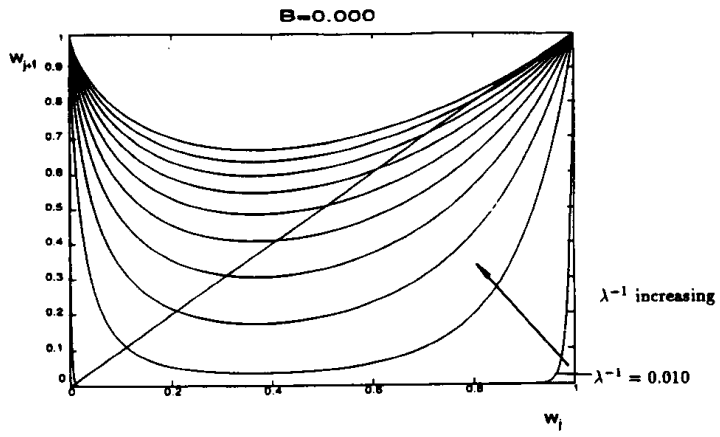

Figure 4(a): Graph of $f\left(w_{j}, \lambda\right)$ for $\beta=0$

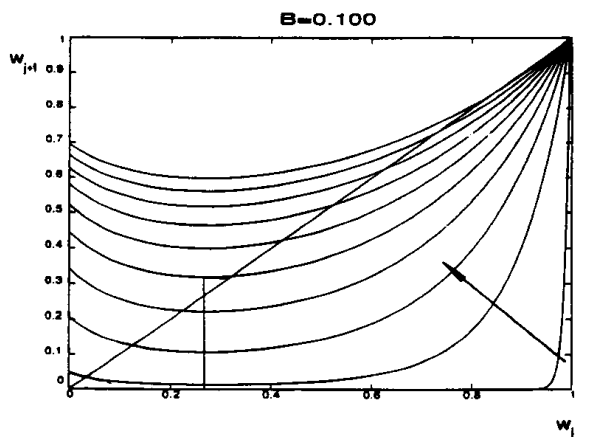

Figure 4(b): Graph of $f\left(w_{j}, \lambda\right)$ for $\beta=0.1$ computed, as in [2]:

$$
\bar{w}=1,1 / \lambda
$$

Thus for $0<\lambda^{-1}<1$ the map has two fixed points in $(0,1]$. When $\lambda^{-1}>1$, the map has a fixed point at $\bar{w}>1$, which is physically unrealizable. Geometrically, the fixed points of the map correspond to intersections of the graph of the map with the $45^{\circ}$ line passing through the origin [3]. The stability of the fixed points is determined by the slope of the graph $w_{j+1}=f\left(w_{j} ; \lambda\right)$ at the fixed points [3]. $f^{\prime}(\bar{w}) \mid<1$ implies that the fixed point $\bar{w}$ is stable. $\left|f^{\prime}(\bar{w})\right|>1$ implies that the fixed point $\bar{w}$ is unstable. For $\lambda<1$ (which is physically meaningless), the fixed point $\bar{w}=1$ is stable and the point $A^{\prime}(\bar{w}=1 / \lambda)$ in Figure $5(a)$ is unstable. For $\lambda>1$ the $\bar{w}=1$ fixed point becomes unstable and point $A^{\prime \prime}(\bar{w}=1 / \lambda<1)$ in Figure $5(\mathrm{a})$ becomes stable. Thus at $\lambda^{-1}=1$ the map (15) undergoes a Transcritical Bifurcation.

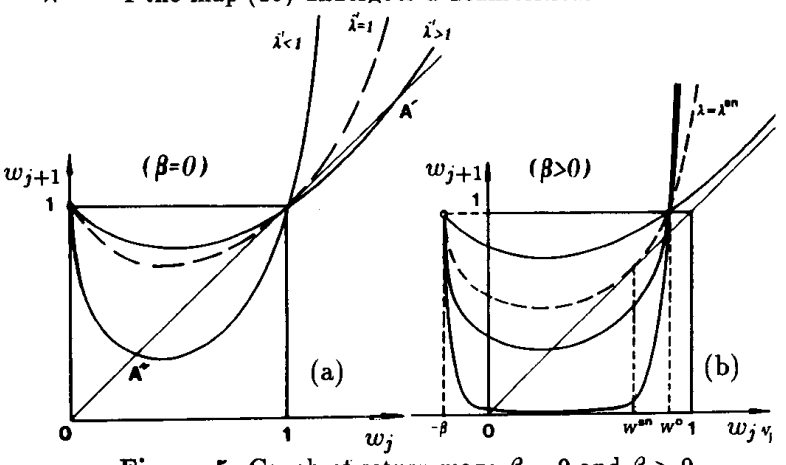

Figure 5: Graph of return map: $\beta=0$ and $\beta>0$. 
Initially, for $\lambda^{-1}$ close to, but less than, 1 , point $A^{\prime \prime}$ is stable. However, at $\bar{w}=\lambda^{-1}=1 / e^{2}, f^{\prime}(\bar{w})=-1$. The fixed point becomes unstable, and at $\lambda^{-1}=1 / e^{2}$, a period doubling bifurcation occurs. To geometrically realize what happens, consider the second iterate of the map:

$$
f^{2}\left(w_{j}\right)=f\left(f\left(w_{j}\right)\right)
$$
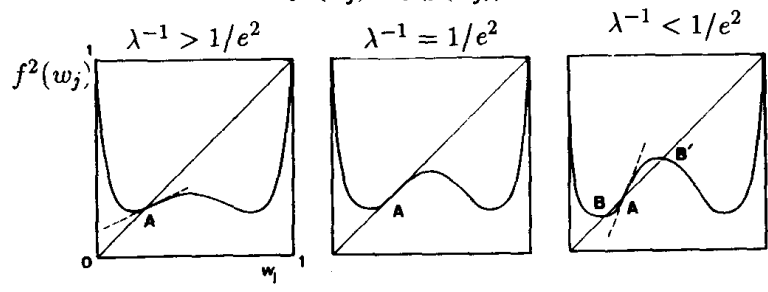

Figure 6: Period Doubling Bifurcation

As $\lambda$ is varied, the graph of the map varies as shown in Figure 6. All intersections of the $45^{\circ}$-line with the graph correspond to period-2 fixed points of the map. Point $A$ in Figure 6 is a degenerate period-2 orbit (it is actually the period-1 fixed point). As $\lambda^{-1}$ decreases, the fixed point $A$ loses stability and a stable pair of period-2 points is generated (stable, for $\lambda^{-1}$ sufficiently close to $1 / e^{2}$ ).

This newly generated stable pair will experience a further period doubling bifurcation as $\lambda^{-1}$ decreases, in which it will lose stability, giving rise at the same time to a period 4 stable periodic orbit, which itself will experience the period doubling phenomenon as $\lambda^{-1}$ is further decreased. The phenomenon just described will give rise to a period doubling cascade of periodic orbits of period $2^{n}$ with $n$ arbitrarily large. In fact, this period doubling situation is recognized as one of the possible routes to chaos $[3,4]$.

\subsection{The Bifurcation Diagram for $\beta=0$}

This simple 1-dimensional map may posses very complicated dynamics, depending on the value of the parameter $\lambda^{-1}$. As we examine higher and higher iterates of the map, explicit analytical computations become increasingly more difficult to perform. To study the dynamics of the map, we resort to numerical computation of its bifurcation diagram. The details of the numerical construction can be found in [7].

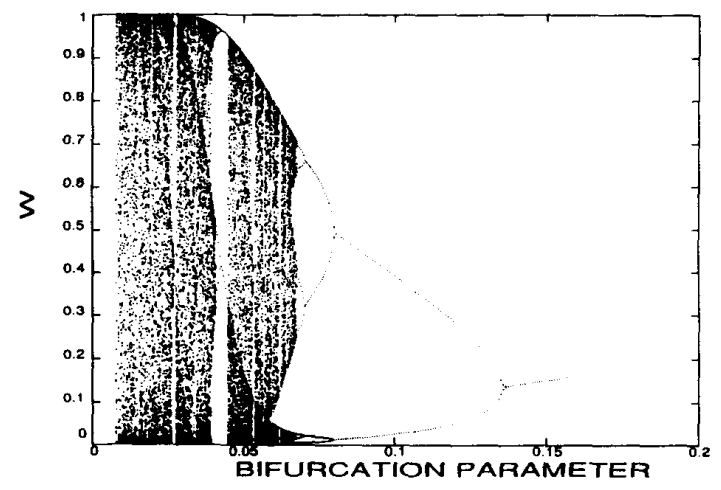

Figure 7: Bifurcation Diagram for $\beta=0$

Figure 7 shows the bifurcation diagram of this system: a plot of the fixed points, $\bar{w}$, vs. bifurcation parameter, $\lambda^{-1}$. The general form of the bifurcation diagram is typical of a general class of unimodal 1-dimensional maps [4]. The period doubling cascade starts at $\lambda^{-1}=1 / e^{2}$. Subsequent period doubling bifurcations leading to stable period $4,8, \cdots$ orbits can clearly be detected. Feigenbaum [5] showed that there is a universal feature of this period doubling sequence for continuous 1-dimensional maps with a quadratic extremum:

$$
\lim _{i \rightarrow \infty} \frac{\left|\lambda_{i}-\lambda_{i-1}\right|}{\left|\lambda_{i+1}-\lambda_{i}\right|}=\delta=4.669201
$$

where $\lambda_{i}$ is the value of the bifurcation parameter for which the periodic orbit of period $2^{i+1}$ is generated. Thus, the relative position of subsequent period doubling bifurcations (for maps with a quadratic extremum) follows a universal pattern which does not depend on the particular form of the map. Furthermore, the period doubling sequence converges to a definite limit at which the final orbit of "period $2^{\infty}$ " is generated. This occurs for a value of the bifurcation parameter:

$$
\lambda_{\infty} \cong \frac{\mathcal{F} \lambda_{i+1}-\lambda_{i}}{\mathcal{F}-1}
$$

where $\mathcal{F}=4.669202 \cdots$ Numerically computed values, derived from Figure 7, confirm the convergence of the ratio (18) to the Feigenbaum constant. From (19), we compute $\lambda_{\infty}^{-1} \simeq 0.06810$.

A second universal constant for maps with quadratic extremum is:

$$
\lim _{i \rightarrow \infty} \frac{\left|w_{i}-w_{i-1}\right|}{\left|w_{i+1}-w_{i}\right|}=\alpha=2.502907875 \cdots
$$

where $w_{i}$ is the value of $w$ at which the period doubling leading to the $2^{i+1}$ orbit is generated. Thus distances on the $w$-axis between points on the attractor are also reduced by a universal scale factor. Again, numerical values of $w_{i}$ from Figure 7 confirm the convergence of the ratio (20) to its theoretical value, and the value of $w_{j}$ for the " $2^{\infty}$ orbit" was computed as $w_{j}=0.77277$.

Until the period doubling cascade has reached its limit, the attractor consists only of even period orbits. After the accumulation value of $\lambda^{-1}$, we expect to find odd period orbits, generated by tangent (saddle-node) bifurcations [4], but the complete story of what happens after the initial period doubling cascade is not yet fully known, and is a current research topic [8].

After the accumulation value of $\lambda^{-1}=0.06810$, the system has an infinity of periodic orbits, most of which are unstable. Lowering still the value of $\lambda^{-1}$, the "strange attractor" appears and the orbits of the map seem to wander randomly inside the attractor without any particular pattern. It must be noted that it is not still clear what a "strange attractor" is (periodic orbits of very large period or the result of numerical instabilities, or $\cdots$ ). However, an interesting feature of the attractor are the windows of periodicity observed in Figure 7. The largest such window appears at $\lambda^{-1} \simeq 0.04494$ where a pair of period three orbits is generated by a tangent (saddle-node) bifurcation[3]. A further discussion of the windows of periodicity are given in [7].

Summarizing, as we increase the thrust applied to the systems for fixed spring constant $\eta_{1}$ and lift-off height $x_{l_{0}}$, the stable period-one hops become unstable, giving increasingly more complicated dynamics. However, as we will see in the next section, the assumption of impulsive thrust, $\delta_{t}=0$, is a limitation in our model. The bifurcation diagram of Figure 7 is "structurally unstable" in the sense that the slightest increase of the value of $\beta$ will result in a qualitatively different bifurcation diagram. 


\subsection{The Case $\beta>0$}

We will now examine (14) for $\delta_{t}>0$. We first give a few preliminary remarks concerning the form of (14) for fixed $\beta$ :

i) $w_{j+1}=1$ for $w_{j}=w^{o}$, where $w^{o}$ is the solution of the transcendental equation:

$$
\frac{\beta}{\beta+w^{o}}=\ln \left(\beta+w^{o}\right)
$$

For $1>w_{j}>w^{\circ}$, the iterates of the map are greater than one and this results in physically unrealisable solutions for the motion of the robot.

ii) The critical point of the map occurs at $w_{j}=w^{c}=e^{-1}-\beta$.

iii) Comparing Figures 5(a) and 5(b), we see that for arbitrarily small values of $\beta$, the singularity of the maps in Figure 5(b) is displaced to negative values of $w_{j}$. The removal of the singularity from $w_{j}=0$, when $\beta>0$, introduces a dynamical behavior quantitatively different from that of $\beta=0$ map.

The fixed points of the map are solutions of the equation:

$$
\bar{w}=e^{\{-\lambda \beta+(\lambda \beta+\lambda \bar{w}) \ln (\beta+\bar{w})\}}
$$

Depending on the values of $\lambda$ and $\beta$, equation (22) may not posses real solutions, i.e. it might be possible that no fixed points exist for the map. We can see in Figure 5(b) that for fixed $\beta$, two fixed points are generated in a saddle node bifurcation. To compute the values of $\lambda$ and $w$ for a saddle node bifurcation, equation (22) should be solved simultaneously with the condition that the slope of the graph at the fixed point is +1 . These conditions lead to the set of transcendental equations:

$$
\begin{aligned}
w^{s n} & =e^{\left\{-\lambda^{s n} \beta+\left(\lambda^{s n} \beta+\lambda^{s n} w^{s n}\right) \ln \left(\beta+w^{s n}\right)\right\}} \\
\ln \left(\beta+w^{s n}\right) & =\frac{1}{\lambda^{s n} w^{s n}}-1
\end{aligned}
$$

We will not solve (23) since the attractor of the map can be found by numerical means as above. Note however that for $\lambda<\lambda^{s n}$ no fixed points exist for the map and thus the robot can not perform any form of periodic hops.

As $\lambda$ is increased from the value corresponding to the initial saddle node bifurcation, the stable fixed point undergoes a period doubling bifurcation and thus initiates a period doubling cascade similar to the one observed for the case $\beta=0$. For fixed $\beta$, the values of $w$ and $\lambda$ necessary for period doubling bifurcations are determined from the following simultaneous set of equations:

$$
\begin{aligned}
w^{p d} & =e^{\left\{-\lambda^{p d} \beta+\left(\lambda^{p d} \beta+\lambda^{p d} w^{p d}\right) \ln \left(\beta+w^{p d}\right)\right\}} \\
\ln \left(\beta+w^{p d}\right) & =\frac{-1}{\lambda^{p d} w^{p d}}-1
\end{aligned}
$$

Equations (24) can be solved to find the bifurcation values $\lambda=$ $\lambda^{p d}(\beta)$ for different values of $\beta$. This is the upper curve shown in Figure 8 [6]. Note that all the complicated (and exciting) dynamics of the map occur in the region of the $\beta$ - $\lambda$ plane enclosed by this curve. The other curves in this figure bound the period 4 and chaotic regions in the $\beta-\lambda$ plane. There is a large amount of structure inside the period 4 curve which is omitted in this diagram for simplicity [7][8].

For values of $\beta$ sufficiently small, the map posses the initial pe-

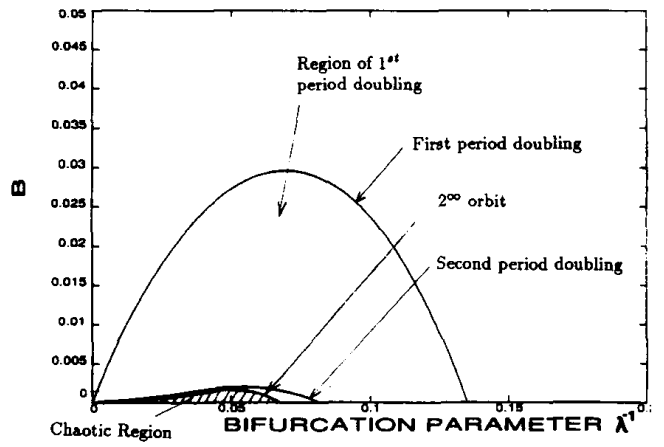

Figure 8: Period Doubling Boundary in $\beta-\lambda$ Plane

riod doubling cascade to chaos and eventually develops a "strange attractor" where motion is unpredictable and very sensitive to initial conditions. However, an exclusive feature of this family of the $\beta>0$ mappings is that the whole structure of the strange attractor ultimately degenerates in a reverse period doubling sequence. As $\lambda^{-1} \rightarrow 0$, the attractor collapses to the initial period one unstable orbit, which regains its initial stability. Note that as we increase $\beta$, the dynamics of the map become less and less complicated, and that above the value of $\beta \simeq 0.0285$ not even a period doubling bifurcation is possible. The series of bifurcation diagrams in Figure 9 demonstrate the slow destruction of the strange attractor as $\beta$ is increased. This generation and subsequent collapse of the strange attractor cannot be found in the simplified map, $\beta=0$. Consequently, increasing the time of thrust, $\delta_{t}$, stabilizes the robot hopping motions primarily by causing the collapse of the strange attractor. If $\delta_{t}$ is chosen such that $\beta>0.0285$, the robot exhibits only period one stable hops, irrespective of the value of $\lambda$. A more complete series of bifurcation diagrams can be found in [7].

In Figure 10, the slope of the map at the fixed point is plotted against the bifurcation parameter $\lambda^{-1}$ for selected values of $\beta$. For $\beta=0$, the slope at the fixed point is continuously decreasing, giving rise to the one-way-route-to-chaos, observed in the bifurcation diagram of Figure 7. However, for $\beta$ arbitrarily small, but not zero, the singularity of the map is removed from $w_{j}=0$ and hence the slope of the map at the fixed points reaches a minimum and then increases to the value $0\left(\right.$ at $\left.\lambda^{-1}=0\right)$. Thus, the strange attractor is forced to collapse as the slope of the map at the fixed point increases from its minimum value. Note that if the minimum of the slope is greater than -1 , then not even a period doubling bifurcation is possible.

\section{Numerical Simulations}

Figures 11(a) and (b) give the simulation results of hopping motion with $\beta=0.001$, corresponding to the bifurcation diagram in Figure 9(d). The actual physical parameters of the system are: $x_{l_{0}}=1, \tau=55.555$, and $\delta_{t}=0.006$. As the stiffness of the spring $\eta_{1}$ varies, the parameter $\lambda^{-1}$ varies and this results in changes of the attractor of the system according to the Figure 9 (b). 11(a) shows period-2 hopping motions $\left(\lambda^{-1}=0.009\right)$, while 11(b) shows choatic hopping motions $(\lambda=0.05)$. Note that $x(t)$ approaches very close to zero in some of the stances, which may be inconsistent with the physical capabilities of a real physical hopping robot, as actuator saturation or other non-linearities not included in the model might occur. A more complete series of simulated hopping motions showing motions of different periods can be found in [7]. 


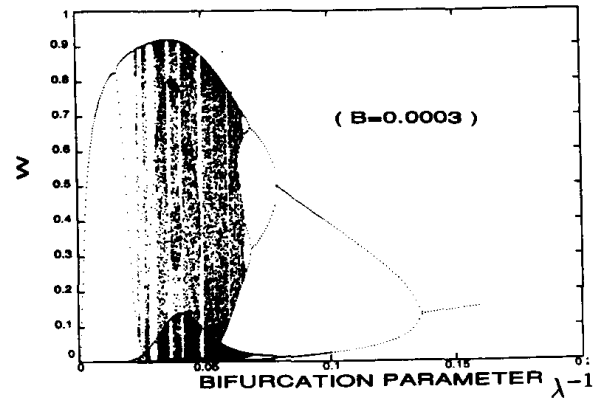

(a)
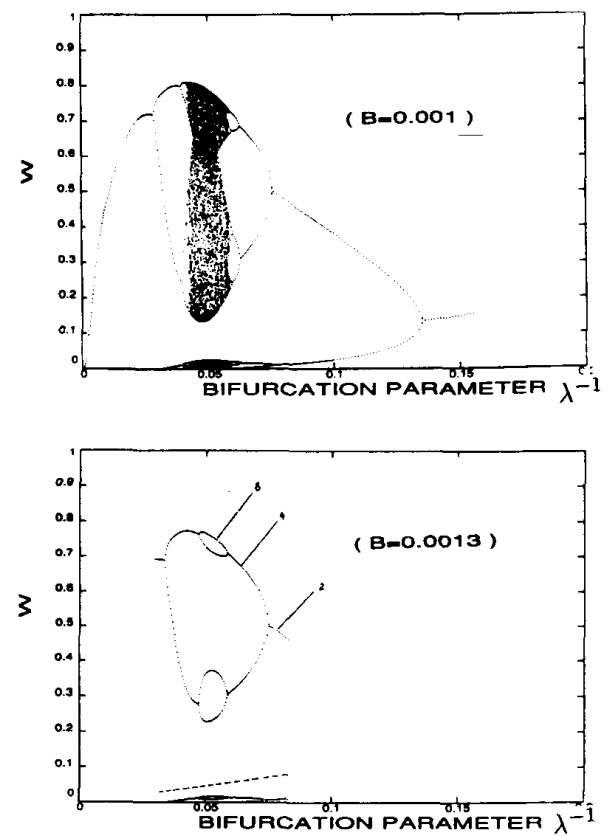

Figure 8: Bifurcation Diagrams for $\beta>0$

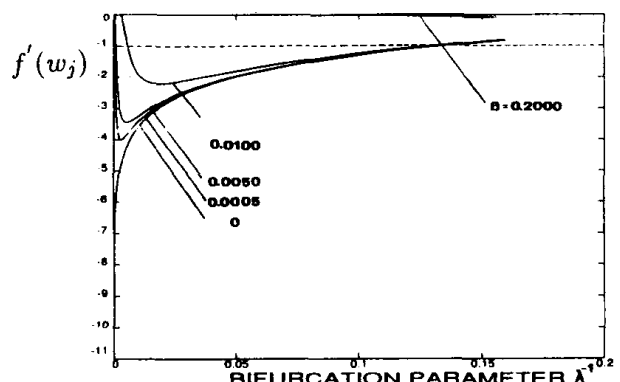

Figure 10: Slope of the Return Map at Fixed Points 6. Discussion

This work investigated the dynamics of a simplified hopping robot. We did this by using and extending the Koditschek/Bühler model, which although simple and incomplete (as all models are!), is hoped to reflect certain dynamic features of the actual hopping systems. Of course, the validity of representing com-
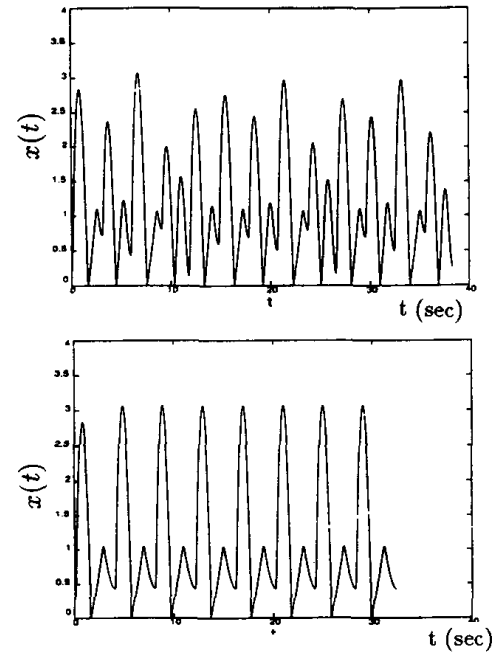

Figure 11: Simulated Vertical Hopping Trajectories

plicated mechanisms with simplified models can only be verified with physical experiements. An interesting feature of this model is that after the attractor reaches a certain point of complexity (becomes a Strange Attractor), it self-destructs in a reverse doubling sequence, ultimately collapsing to a period-1 orbit. In addition, the structure of the attractor becomes less and less complicated for increasing $\delta_{t}$. More complete details of why this trend occurs can be found in [8]. If the dynamics of this models can capture certain dynamical characteristics of actual hopping systems, then the model can be used to identify the basic physical parameters controlling hopping dynamics and furthermore predict dynamic performance as these parameters are varied. This paper showed that that in addition to the parameter $\lambda$, the thrust parameter $\beta$ is of great importance since it essentially controls the complexity of the robot's attractor.

\section{References}

[1] M. H. Raibert, Legged Robots that Balance, The MIT Press, Cambridge, Mass., 1986.

[2] D. E. Koditschek and M. Bühler, "Analysis of a Simplified Hopping Robot," Report no. 8804, Dept. of Electrical Engineering, Yale University, 1988.

[3] J. Guckenheimer and P. Holmes, Nonlinear Oscillations, Dynamical Systems and Bifurcations of Vector Fields, Springer Verlag, New York, 1983.

[4] R. Devaney, An Introduction to Chaotic Dynamical Systems, Addison-Wesley Publishing Co., New York, 1987.

[5] M. J. Feigenbaum, "Quantitative Universality for a Class of Nonlinear Transformations," J. of Stat. Physics, vol. 19, no. 1, 1978, pp. 25-185.

[6] P. Holmes, personal communication.

[7] J.W. Burdick, A.F. Vakakis, and T.K. Caughey, "Periodic Orbits and a Strange-Strange Attractor In the Dynamics of a Simplified Hopping Robot," Robotics and Mechanical Systems Report no. RMS-89-01, School of Engineering and Applied Science, California Institute of Technology, August, 1989.

[8] A.F. Vakakis, J.W. Burdick, "Study of a One-Dimensional Map Describing the Dynamicsl of a Hopping Robot," Robotics and Mechanical Systems Report no. RMS-90-01, School of Engineering and Applied Science, California Institute of Technology, in preparation. 